\title{
A quality improvement project on discharge summaries completion in a nephrology ward
}

\author{
Author: Aruna Arjunan*
}

\section{Introduction}

Discharge summary is an essential part of patient care and is a patient safety issue if not completed on time. The NHS England Standard Contract guidance insists that discharge summaries should be completed and sent to general practitioner within 24 hours of discharge. Dorset County Hospital aims for $98 \%$ discharge summaries to be completed within 24 hours. The aim of the project was to improve the 24 -hour electronic discharge summary completion rates in the renal ward, from a baseline of $30 \%$ to $60 \%$ in the 3-month period from February 2018 to May 2018.

\section{Methods and materials}

\section{Main challenges}

The completion rates of the renal ward were lower than the rest of the hospital due to various reasons. An unfilled ward clerk position had resulted in difficulties accessing patient records in a timely manner. As day case procedures were archived using unique codes, there was a misconception that they don't require discharge summaries. Poor staffing levels, lack of administration time in a busy placement and lack of insight into the importance of discharge summaries had resulted in a general lack of ownership within the team.

\section{Interventions (Plan, Do)}

The electronic discharge summary (EDS) system had an 'inactive' option of creating the list of incomplete discharge summaries for any ward. Arrangements were made through the information team to electronically update this list twice daily. A new routine of reviewing this list with the team, daily after handover was implemented. A team member was identified as the responsible person to clear the incomplete summaries. The team was educated on the impact on patient safety through patient stories, which improved their engagement. Registrars completed discharge summaries for all day case procedures. Temporary ward clerks helped to clear the backlogs.

\section{Results and discussion (Study)}

The percentage of completed discharge summaries within 24 hours and within 1 month of discharge were measured. Data collection

Author: Dorset County Hospitals Foundation Trust, UK ${ }^{*} \mathrm{RCP}$ chief registrar

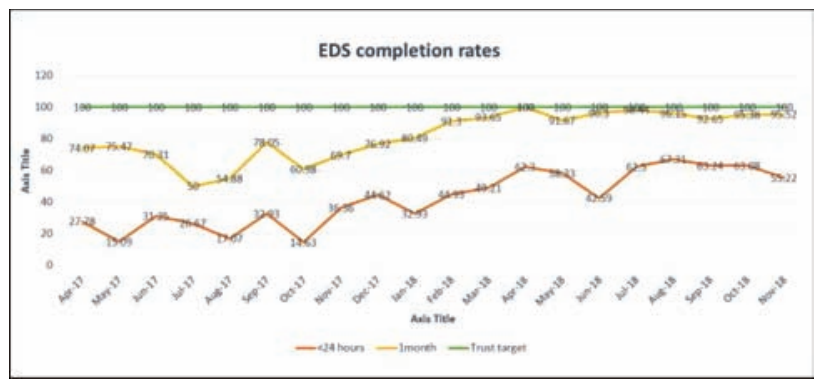

Fig 1. EDS completion rates after the QI project in comparison with baseline

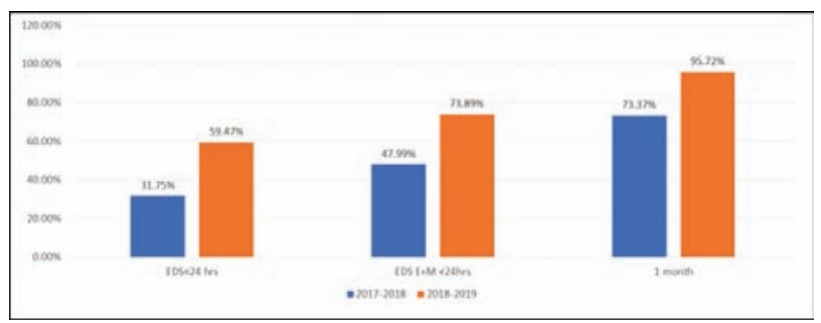

Fig 2. EDS completion rates before and after the QI project

was $100 \%$ complete. Following the QI interventions, the 24-hour discharge summaries completion rates had doubled (see charts 1,2). The monthly discharge summaries completion rates increased from $73.37 \%$ to $95.72 \%$. The quality of discharge summaries was not compromised, and the summary content followed the national requirements.

\section{Next steps (Act)}

Although the improvements were sustained, the completion rates did not increase exponentially, but plateaued after the initial rise. Some potential reasons are weekend discharges and deceased patients. Further exploration of potential reasons and repeat PDSA cycles are required for further improvement.

\section{Conclusion}

All the progress achieved in this project was sustained due to the involvement of the entire team. The team who once viewed 
discharge summary as irrelevant to patient care and as a timeconsuming task of no learning value, changed their attitude once they realised the importance of it. The key learning points from this project are 'communication is the key to implement change' and 'patient stories are powerful'.

\section{Reference}

1 Singh I. Guidance on the NHS Standard Contract requirements on discharge summaries and clinic letters and on interoperability of clinical IT systems. NHS England, 2018. 